\title{
Web al Servicio de la Anatomía: Relato de una Experiencia Docente
}

\author{
Web Service Anatomy: Report of an Educational Experience
}

Emilio Farfán C."; Daniela Schneeberger L."; Jovita Besa H."; Guillermo Salgado A.. \& Oscar Inzunza H."

FARFÁN, C. E.; SCHNEEBERGER, L. D.; BESA, H. J.; SALGADO, A. G. \& INZUNZA, H. O. Web al servicio de la anatomía: relato de una experiencia docente. Int. J. Morphol., 34(1):136-142, 2016.

RESUMEN: La permanente actualización de los programas curriculares de educación médica ha generado discusión respecto a los modelos de aprendizaje utilizados para las ciencias morfológicas, tema muy en boga hoy en día, debatido por profesores de morfología y especialistas en docencia. El presente trabajo da a conocer el modelo de enseñanza morfológica utilizado en el departamento de Anatomía Normal de la Pontificia Universidad Católica de Chile, el cual se ha desarrollado consecuentemente con los cambios curriculares realizados por la escuela de medicina, logrando adaptarse a los nuevos tiempos y nuevas tecnologías. Este modelo de aprendizaje constructivista se basa en estudio guiado y auto dirigido con material web creado especialmente para cada curso, privilegiando el trabajo práctico en pabellón y complementándolo con recursos tecnológicos, como evaluaciones teóricas en computadores y evaluaciones prácticas utilizando tablets en el pabellón entre otras. Todo este trabajo bajo una misma plataforma web. Estos cambios han mejorado la interacción docente-alumno y facilitado el trabajo académico. Los resultados de este modelo han sido evaluados positivamente por el equipo docente y permiten concluir que la inclusión de nuevas tecnologías como complemento para la enseñanza de la morfología son un real aporte, siendo bien recibidas por los estudiantes.

PALABRAS CLAVE: Anatomía; Educación; Web.

\section{INTRODUCCIÓN}

La educación en las ciencias anatómicas ha sufrido varios cambios a lo largo de la última década (Drake et al., 2009), ha debido adaptarse a los cambios curriculares según la época, quedando esto descrito en las transformaciones vividas en las "reformas de la educación médica" y las "reformas de enseñanza de la anatomía" (Hildebrandt, 2010), incluso viendo amenazada su continuidad por reducciones en extensión y contenidos (Jones \& Harris, 1998). Respecto a ello hay voces que sugieren que la integración de nuevas modalidades de enseñanza y la tecnología moderna constituyen una manera de fomentar el interés y la retención de los conocimientos anatómicos y su relevancia clínica (Turney, 2007).

Frente a esta situación y observando el desarrollo tecnológico las ciencias morfológicas se encuentran en una excelente oportunidad de reinventarse. Por ejemplo, evaluando la real utilidad de una clase expositiva, existe evidencia que reconoce mejoras significativas de rendimiento aplicando el aprendizaje auto dirigido por sobre clases expositivas (Vasan et al., 2009); además de una favorable percepción por parte de los estudiantes. Asimismo, es evidente que ha habido un auge en cuanto a la utilización de nuevas tecnologías para la enseñanza de la anatomía; resultados de trabajos sobre el uso de software docente en los cursos de anatomía muestran inequívocamente que la utilización de imágenes de preparaciones anatómicas tratadas en forma de animaciones, tienen un impacto real en el aprendizaje práctico de anatomía humana. Este impacto se traduce en un incremento estadísticamente significativo de las calificaciones obtenidas por los estudiantes en las evaluaciones prácticas (Inzunza \& Bravo, 1999). Además, ha quedado demostrado que la utilización de imágenes anatómicas computacionales representan un apoyo importante a las actividades prácticas, mejorando el rendimiento en el reconocimiento de imágenes en computador o imágenes radiológicas (Inzunza et al., 2003). En la misma línea en Hungría se ha dado a conocer un curso de anatomía macroscópica asistido por un computador, centrado fundamentalmente en imágenes asociadas a casos clínicos, orientado a estudiantes internacionales que no dominaran el idioma húngaro, consiguiendo mejoras significativas respecto a quienes no utilizaron el software y con ello

* Departamento de Anatomía, Escuela de Medicina, Pontificia Universidad Católica de Chile, Santiago, Chile. 
superando la barrera del lenguaje (Kish et al., 2013). En el último tiempo también se ha comentado sobre la realidad virtual, cuyos resultados no han encontrado evidencia de mejoras significativas en el aprendizaje (Garg et al., 2002). Así mismo, no hay pruebas suficientes para demostrar que estos recursos tecnológicos pueden sustituir a los métodos tradicionales en enseñanza de la anatomía, además se deben realizar investigaciones para determinar cómo utilizar estas tecnologías junto con nuevos métodos de enseñanza o cómo su uso puede ser integrado en el plan de estudios de anatomía actual (Tam et al., 2009). Respecto al incremento de tecnologías móviles como tablets y su rol cada vez más protagónico en la educación médica a través de sus aplicaciones, hay evidencia que avala su utilidad para el aprendizaje cuando se complementa con otros métodos existentes (Lewis et al., 2014); coincidiendo esto con experiencias que han descrito que el aprendizaje asistido con tablets logra los objetivos de instrucción, y mejora la eficacia y eficiencia educativa de la disección (Mayfield et al., 2013).

Estudios concluyen que el uso de la computación está cambiando el rol del profesor, pues no sólo se dedicará a dictar clases, sino que coordinará un trabajo grupal de responsabilidad compartida. En este sentido, buscar, comprender, evaluar y seleccionar información obligaría a usar el razonamiento sobre la memoria (Lacruz et al., 2009). Es decir, los sistemas de aprendizaje activo abren nuevos horizontes en el ambiente de educación virtual (Sheremetov \& Vladimir, 2002). De esta manera el uso de tecnologías como plataformas web, fundamentalmente lo que hacen es facilitar el acceso al material auto instructivo, brindando a estudiantes y docentes una serie de servicios, tales como bibliografía, foros, correo, etc., en un entorno reducido, accesible mediante nombre de usuario y contraseña, y con un funcionamiento uniforme (Dans, 2009).

Conscientes de esta realidad nuestro equipo docente ha dado pasos en pos del desarrollo tecnológico, y así como en la década del 90 se creó el material pedagógico web para los estudiantes, y con ello dando inicio al aprendizaje constructivista. Ahora se integraron a este sistema las evaluaciones en línea, iniciándose el año 2012 un proceso paulatino de traspaso de todas las evaluaciones en papel a las pantallas.

\section{MATERIAL Y METODO}

Docencia. El Departamento de Anatomía Humana nace en el año 1930, junto con la fundación de la Escuela de Medicina de la Pontificia Universidad Católica de Chile. En 1954 nuevos planes de estudio se ponen en marcha en la Escuela de Medicina producto de la necesaria adecuación que los estudios médicos debían tener de acuerdo a la época y el avance de la ciencia. Como consecuencia de ello, Anatomía vio reducido su curso de dos años a un año. Pasó un largo tiempo de docencia tradicional, hasta que en 1994 el curso utiliza el Software A.D.A.M. como parte del proceso educativo, además de la base de imágenes Slice of Life. En los años siguientes se desarrollan consecutivos proyectos docentes en esta línea. Como resultado de lo anterior, en la actualidad los cursos de pregrado del Departamento de Anatomía para Medicina, Enfermería, Odontología, Kinesiología, Nutrición y Fonoaudiología cuentan cada uno con páginas web de apoyo docente, las cuales tienen una gran demanda tanto de los alumnos de la Facultad de Medicina de nuestra casa de estudios, como de otras universidades. Así, de a poco fue cambiando el sistema tradicional de clases expositivas y actividades prácticas, involucrando activamente a los estudiantes, aplicando la teoría constructivista del aprendizaje y elaborando un trabajo que se basa en el aprendizaje auto dirigido (Bergman et al., 2013). Para ello se les asigna lectura previa guiada para cada actividad práctica, según el texto que corresponda, y una página web especialmente construida para cada curso. Con esta información previa, los alumnos en grupos pequeños (4 a 6) se enfrentan a las preparaciones cadavéricas, procediendo a reconocer las estructuras y resolver preguntas morfológicas y de aplicación clínica señaladas en los objetivos de cada actividad. Esta experiencia es similar team-based learning (TBL) descrito por otros autores (Huitt et al., 2014; Michaelsen \& Richards, 2005). Este paradigma docente privilegia el trabajo en pabellón, destinando aproximadamente el $75 \%$ de sus horas docentes directas a las actividades prácticas y un $25 \%$ a clases expositivas que son esencialmente clínicas y aplicativas, realizadas por especialistas de acuerdo los contenidos. De este modo, se favorece el contacto con material cadavérico, actividad muy valorada por los estudiantes (Barrovecchio et al., 1998; Johnson, 2002; Azer \& Eizenberg, 2007; Aziz, et al., 2002), y más aún cuando es con orientación clínica (Clough \& Lehr, 1996). Esta metodología en conjunto con la utilización de softwares anatómicos han permitido un mejor acercamiento a la situación de reconocimiento de elementos anatómicos en el cadáver, realidad sobre la cual el alumno deberá actuar (Inzunza \& Bravo, 2002). De esta manera y con el pasar de los años se renovó el sistema de aprendizaje, por lo que el siguiente paso fue la renovación del sistema evaluativo.

Año 2012. Hasta el año 2012 todas las evaluaciones que realizaba el departamento de anatomía normal se hacían en papel, esto incluye evaluaciones teóricas, practicas y controles de entrada. Desde el segundo semestre de ese año el departamento decide comenzar a utilizar la plataforma web que utiliza la Pontificia Universidad Católica llamada 
webcurso (basado en proyecto Sakai), la cual permite ingresar a través de usuario y contraseña a los distintos cursos, realizar evaluaciones de todo tipo, llevar planillas de notas, mensajería interna, foros de discusión y estadísticas de desempeño entre otros.

La iniciativa para utilizar esta plataforma se vio impulsada por varios motivos, entre ellos están: el incremento del número de cursos y el número de alumnos, el manejo de las nuevas tecnologías por parte de docentes y estudiantes, la eficiencia que entrega éste sistema de trabajo, la infraestructura disponible, el significativo ahorro económico al no gastar en papel y tinta lo que redunda en una contribución ecológica.

Año 2013. El cambio fue paulatino y cronológico, lo primero que se hizo fue una capacitación a los docentes del departamento de anatomía, esta se realizó en enero del año 2013, donde se les enseñó a realizar distintos formatos de evaluaciones de acuerdo a las necesidades del departamento. Inicialmente se decidió realizar a través de webcurso los controles de entrada para todos los cursos y además, realizar las evaluaciones teóricas sólo con el curso más pequeño (en número de alumnos y horas por semana), de este modo todas las evaluaciones prácticas siguieron siendo en papel.
Controles de entrada. Se traspasaron a webcurso los controles de entrada, manteniendo el formato de preguntas de correlación y la duración (5 min). Estas evaluaciones se podían realizar desde cualquier lugar, sólo se requería tener un dispositivo electrónico (Computador, Tablet o Smartphone) y acceso a internet. Sin embargo, se tomo la precaución de reservar para todos los cursos las salas de computadores, en sus respectivos horarios, para que pudieran rendir allí los controles de entrada antes de que ingresaran al pabellón de anatomía. Para la anécdota queda la historia de una alumna que debió viajar al extranjero y nos envió un correo agradecida del sistema porque pudo realizar su control correspondiente en un Starbucks desde Nueva York.

Acertijo morfológico. Reconociendo las nuevas posibilidades que nos ofreció la plataforma web se creó el "Acertijo Morfológico", el cual consiste en una evaluación donde se publica una fotografía de nuestras disecciones y los alumnos deben identificar una serie de elementos y contestar en un tiempo de $10 \mathrm{~min}$. Esta actividad se considera como nota de controles de entrada; sin embargo, la diferencia es que se trata de una fotografía rotulada con 7 a 10 estructuras a reconocer, y por lo tanto es un formato distinto, totalmente voluntario y fuera de horario de clases (Fig. 1).

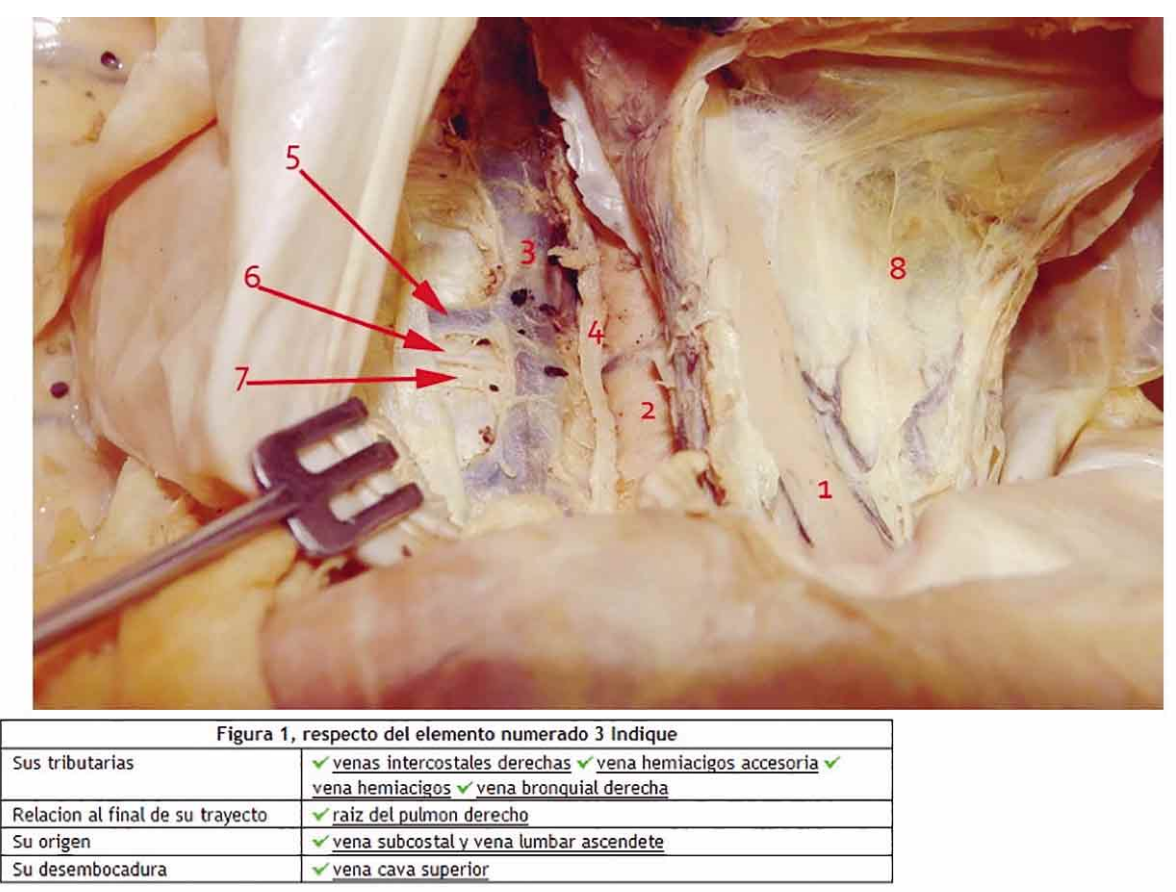

Clave de las respuestas: venas intercostales posteriores / hemiacigos / acigos accesoria|venas bronquiales, venas intercostales posteriores | hemiacigos |acigos accesoria|venas bronquiales, venas intercostales posteriores|hemiacigos $\mid$ acigos accesoria|venas bronquiales, venas intercostales posteriores $\mid$ hemiacigos $\mid$ acigos accesoria| venas bronquiales, pediculo pulmonar derecho | bronquio derecho, venas lumbares|continuidad con venas lumbares, vena cava superior

Fig. 1. Fotografía de mediastino posterior utilizada como "acertijo morfológico" realizado al curso de medicina. Este test puede ser realizado en cualquier lugar y desde cualquier dispositivo electrónico. 
Evaluación teórica. Para estas evaluaciones se mantuvo el formato de preguntas, de alternativas, de correlación y de desarrollo, la innovación respecto al papel es que se pudieron reemplazar los esquemas y dibujos por fotografías morfológicas (fotografías de disecciones, preparaciones histológicas y embriológicas). El lugar físico donde los alumnos rinden estas pruebas son las salas de multimedios de la universidad.

Al finalizar el primer semestre del año 2013 la evaluación de este sistema fue positiva, por lo que se decidió incluir en este proyecto a otro de nuestros cursos con un mayor número de alumnos y más horas, completando así 2 cursos que realizaban sus evaluaciones teóricas a través de webcurso en el segundo semestre de ese año.

Con el excelente resultado obtenido el 2013 es que se decidió que para el año 2014 todos los cursos rendirían sus controles de entrada y evaluaciones teóricas a través de la plataforma web. No conforme con ello, nos propusimos implementar un sistema para poder realizar las evaluaciones prácticas a través de webcurso el año 2014 y con ello abandonar definitivamente el papel.

Año 2014. Con el sistema de evaluación traspasado ya en su mayoría a webcurso es que a fines del año 2013 se ideo el plan de utilizar los recursos de la plataforma web para traspasar las evaluaciones prácticas al mismo sistema, idea que se concretó el año 2014. Para esto se evaluó la capacidad de personas de ambos pabellones de anatomía, la factibilidad de instalar un sistema de conectividad inalámbrica que no colapsara y fuese estable, y la compra de tablets suficientes para realizar evaluaciones en ambos pabellones al mismo tiempo.
Pabellones. Nuestro departamento cuenta con 2 pabellones de anatomía, el pabellón poniente, posee una capacidad para 140 personas y el pabellón oriente una capacidad para 80 personas. Considerando los horarios de clases, el máximo posible de estudiantes que podrían rendir simultáneamente evaluaciones prácticas es de 180 , por lo que ambos pabellones se encontraban en condiciones de soportar esta carga.

Sistema de conectividad inalámbrica. El sistema de conexión a internet es fundamental para el correcto funcionamiento del proceso, por lo que se realizó un trabajo en conjunto con los equipos de redes, conectividad y webcurso para implementarlo. El resultado fue conectividad inalámbrica exclusiva para ambos pabellones, distribuyéndose el internet a través de 7 puntos de acceso con capacidad para 60 dispositivos cada uno, superando la capacidad máxima posible de dispositivos conectados al mismo tiempo (180 vs 420).

Tablets. Paralelo a la instalación del sistema de conectividad inalámbrica se realizó la compra de tablets para realizar las evaluaciones, los requerimientos a considerar fueron la capacidad de conexión, tamaño de pantalla, rapidez del equipo y durabilidad. Una vez escogido el equipo se procedió a comprar 185 tablets, entendiendo que el máximo a utilizar en un mismo momento eran 180 y 5 de repuesto.

Una vez implementado el sistema para las evaluaciones prácticas se agregó en los calendarios de todos los cursos una evaluación práctica formativa a inicio de semestre, que permitiera a los estudiantes conocer el sistema y a la vez probar su funcionalidad (Fig. 2).

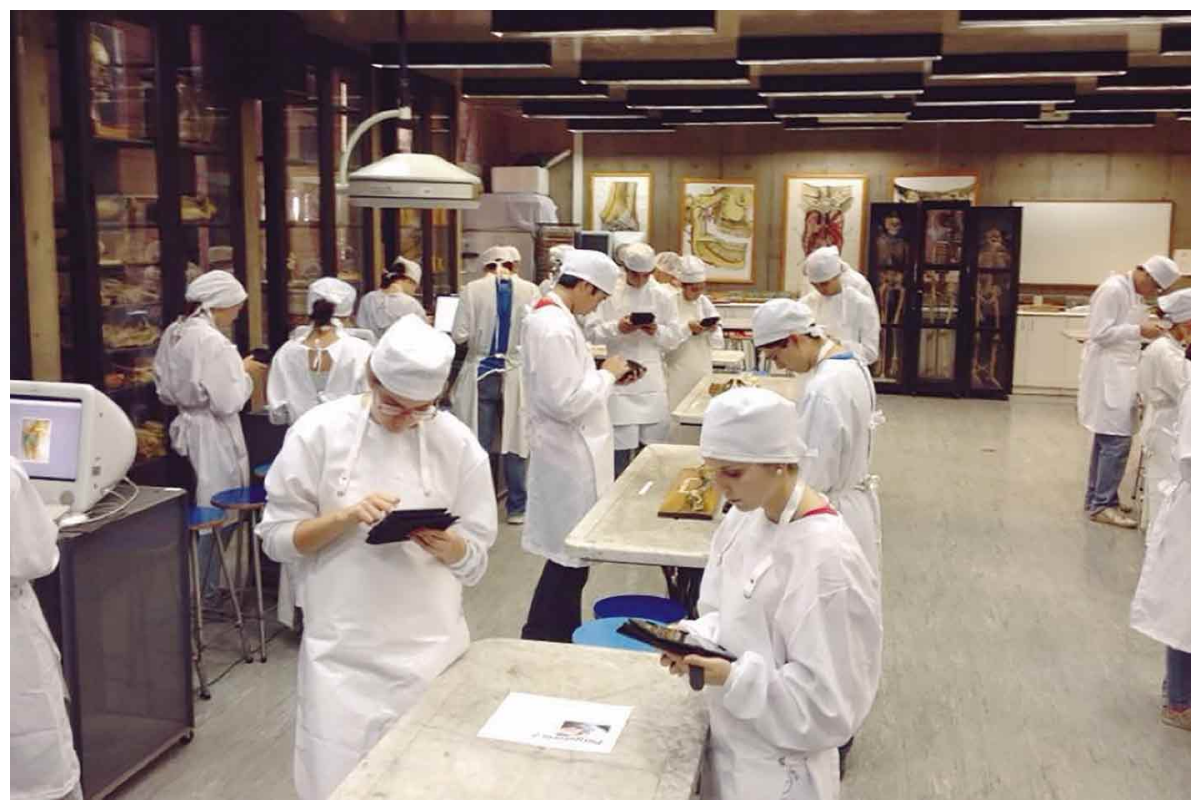

Fig. 2. Fotografía de la primera evaluación práctica (gymkhana) realizada al curso de Medicina, utilizando tablets en lugar de papel. 
El resultado fue exitoso en todas las evaluaciones formativas, lo que permitió traspasar completamente todos los formatos de evaluación utilizados a la plataforma en línea llamada webcurso.

\section{RESULTADOS}

Durante el primer semestre 2014 todas las evaluaciones realizadas por el Departamento de Anatomía Normal de la Pontificia Universidad Católica de Chile fueron realizadas a través de la plataforma webcurso, esto incluye controles de entrada, evaluaciones teóricas y evaluaciones prácticas, que en total suman 126 evaluaciones durante el primer semestre con un promedio 21 evaluaciones por curso (Tabla I).

Es preciso destacar, que al poder contar con el equipo necesario nos fue posible aplicar los controles de entrada, que durante el año 2013 se realizaban desde cualquier equipo y lugar, en nuestros tablets y en los pabellones de anatomía.

\section{DISCUSIÓN}

Sin duda nuestro Departamento de Anatomía ha debido adaptarse a los cambios curriculares transcurridos a lo largo de su historia, sin quedar ajeno a otras situaciones relatadas (Hildebrandt), es por eso que consideramos necesario el estar permanentemente actualizados en cuanto a reformas curriculares y como la anatomía se puede adecuar a ellas, para no ver en riesgo su continuidad (Jones \& Harris). Por lo que consideramos necesario ir en busca de nuevas metodologías de aprendizaje de acuerdo a los tiempos y recursos disponibles (Turney). En este sentido debemos mirar los nuevos horizontes educativos que nos ofrece la educación virtual (Sheremetov \& Vladimir), y el fácil acceso de los estudiantes y docentes a una plataforma web desde cualquier lugar. Además, esta tecnología posibilita mayor interactividad para el alumno, permitiendo un mejor acercamiento a la situación de reconocimiento de elementos anatómicos en el cadáver con el que después el alumno interactuará (Inzunza \& Bravo, 1999). Esta situación se ve muy bien reflejada con los acertijos morfológicos, donde hemos podido constatar la motivación que produce en los estudiantes el desafío de resolver una imagen de alta dificultad, utilizando todas los recursos disponibles, en un tiempo acotado, generando en ellos capacidad de observación, análisis, razonamiento y discusión de ideas entre ellos (Lacruz et al.); todo en pos de su aprendizaje, proceso que tiene un enorme valor educativo.

Otro punto importante es cómo esta tecnología mejora la eficiencia del trabajo docente; por ejemplo, para realizar las evaluaciones en la plataforma webcurso se requiere subir las pautas de cada prueba antes que esta se aplique. En el caso de un control de entrada, cuyo formato es de correlación, una vez que éste haya sido realizado el sistema corrige la evaluación automáticamente y el estudiante puede ver su nota de inmediato. En el caso de una evaluación teórica quedarán inmediatamente corregidos los ítems de término excluido y correlación, y sólo se deberá revisar manualmente la parte de desarrollo. Para las evaluaciones prácticas, la formulación de preguntas debe acotar al mínimo el número de respuestas posibles; de este modo al subir a la plataforma una pauta de evaluación práctica se debe incluir todas las respuestas potenciales, esto asegura la fiabilidad del sistema y reduce la carga del docente dedicada a la corrección de pruebas. Además, el sistema ingresa las notas en una planilla, calculando automáticamente los promedios, siendo estos ejemplos algunos de los tantos servicios entregados por estos sistemas (Dans).

Dentro de las desventajas están el costo económico que implica la implementación de un sistema como éste, el cual sin duda debe ser muy bien desarrollado para que sea exitoso, ya que la más mínima vulnerabilidad conlleva problemas. Otra desventaja es la dependencia tecnológica absoluta que genera. En nuestra experiencia durante el primer semestre del año 2013, cuando las evaluaciones eran en horario de alta congestión cibernética, se presentaron dificultades como el no envío de pruebas o envíos incompletos; este fue uno de los motivos por el cual se creó el equipo multidisciplinario que se dedicó a estudiar, analizar e implementar las soluciones de conectividad más apropiadas. Por lo tanto si se trabaja con los debidos resguardos esta desventaja no existe.

Tabla I. Número alumnos y de evaluaciones por curso.

\begin{tabular}{|c|c|c|c|c|c|c|c|c|}
\hline \multirow{2}{*}{$\begin{array}{l}\text { Curso } \\
\text { (estudiantes) }\end{array}$} & & Medicina & Odontología & Kinesiología & Enfermería & Fonoau diol ogía & Nutrición & Total \\
\hline & & $(n=121)$ & $(n=73)$ & $(n=97)$ & $(n=126)$ & $(n=69)$ & $(n=84)$ & $(n=570$ \\
\hline \multirow[t]{4}{*}{ Evaluación } & Controles & 15 & 15 & 12 & 12 & 10 & 12 & 76 \\
\hline & Ev. Teórica & 3 & 4 & 5 & 5 & 3 & 5 & 25 \\
\hline & Ev. Práctica & 3 & 4 & 5 & 5 & 3 & 5 & 25 \\
\hline & Total & 21 & 23 & 22 & 22 & 16 & 22 & 126 \\
\hline
\end{tabular}


Es claro que los recursos tecnológicos no pueden sustituir por completo al sistema tradicional de enseñanza de la anatomía (Tam et al.), y concordamos con ello, en nuestra experiencia podemos decir que el modelo presentado en este articulo, el cual complementa el estudio auto dirigido, la interacción docente-alumno a través de plataforma web y el mayor énfasis en el trabajo práctico con orientación clínica es lo que nuestros alumnos valoran y agradecen (Barrovecchio et al.; Johnson; Azer \& Eizenberg; Aziz et al.; Clough \& Lehr). De acuerdo a esto, para nosotros lo irremplazable es el trabajo práctico en pabellón, lo que nos hace recordar la siguiente expresión "hasta la década de 1870, los estudiantes de medicina de los Estados Unidos escuchaban mucho, veían poco, y no hacían nada" (Hildebrandt, 2010 citando a Mall 1899).

Implementar este modelo fue un proceso paulatino que se inició en la década del 90 con las páginas web y culmina con evaluaciones prácticas en tablets, sobrellevando un gran esfuerzo inicial en cuanto a capacitación, implementación, coordinación del equipo y transferencia de información. Sin embargo, una vez que el sistema está en curso, facilita enormemente el trabajo docente, reduciendo la carga y agilizando los procesos, es decir, haciendo más eficiente el trabajo académico y lo más importante es que deja en manos del estudiante la responsabilidad de su aprendizaje, transformándolo en el eje en torno al cual gira todo un sistema.

Por último, en nuestro medio es importante destacar que este paradigma docente constituye un aporte en los tema de inclusión y sustentabilidad. Por un lado, apoya las políticas universitarias de inclusión ya que provee a todos los alumnos, independientemente de su origen, las herramientas tecnológicas (software y hardware) para las instancias de estudio y de evaluación de los temas anatómicos. Por otro, aportamos a la sustentabilidad medioambiental al erradicar el uso de tinta y papel para todas las evaluaciones de todos nuestros cursos, 6 cursos de pregrado, con una matrícula anual de más de 500 alumnos, con un promedio de 12 pruebas de entrada, 3 evaluaciones teóricas y 3 evaluaciones prácticas (Fig. 2) por alumno, lo que representa un gasto del orden de 100 resmas de papel y más de 50 cartuchos de tinta negra al año.

\section{CONCLUSIONES}

La anatomía como disciplina ha sabido adaptarse a los cambios curriculares y es nuestra tarea estar permanentemente atento a las oportunidades de renovación, como son hoy los recursos tecnológicos. Los cuales poseen ventajas que en el largo plazo superan ampliamente el esfuerzo que conlleva su implementación, aumentan la eficiencia de los equipos de trabajo y por lo tanto se transforman en una herramienta considerablemente útil y recomendable.

\section{AGRADECIMIENTOS}

Agradecemos al Dr. Hermes Bravo Contreras Profesor Titular del Departamento de Anatomía Normal de la Pontificia Universidad Católica de Chile, quien fue uno de los fundadores de este modelo docente. Así mismo, reconocer a la Dra. Patricia García C. y Srta. Magdalena Rodríguez M. quienes creyeron en este proyecto y gestionaron la adquisición de los recursos computacionales necesarios para la implementación.

FARFÁN, C. E.; SCHNEEBERGER, L. D.; BESA, H. J.; SALGADO, A. G. \& INZUNZA, H. O. Web service anatomy: report of an educational experience. Int. J. Morphol., 34(1):136142,2016

SUMMARY: Continuous curriculum updating of medical education has generated discussion regarding the learning models used for morphological sciences, a topic much in vogue today, discussed by morphology teachers and education specialists. This paper discloses the morphological teaching model used in the Department of Gross Anatomy of the Pontificia Universidad Católica de Chile, which has been consequently developed together with the curriculum changes made by the school of medicine, being able to adapt to current times and new technology. This constructivist learning model is based on a guided and self-directed learning with web-based material specially designed for each course, emphasizing practical work in laboratories and supplemented by technological and theoretical evaluations conducted on computers and practical evaluations using tablets in the laboratories among others. All this work under a single web platform. These changes have improved student-teacher engagement and eased academic workload. The results of this model have been positively evaluated by members of the faculty team and allow to conclude that the inclusion of new technologies as a supplement to teaching morphology are a real contribution, as well as being well received by students.

KEY WORDS: Anatomy; Education; Web.

\section{REFERENCIAS BIBLIOGRÁFICAS}

Azer, S. A. \& Eizenberg, N. Do we need dissection in an integrated problem-based learning medical course? Perceptions of firstand second-year students. Surg. Radiol. Anat., 29(2):173-80, 2007. 
Aziz, M. A.; Mckenzie, J. C.; Wilson, J. S.; Cowie, R. J.; Ayeni, S. A. \& Dunn, B. K. The human cadaver in the age of biomedical informatics. Anat. Rec., 269(1):20-32, 2002.

Barrovecchio, J. C.; Perez, B. \& Bella de Paz, L. Proposals regarding the learning-teaching process in human anatomy. Rev. Chil. Anat., 16(2):219-24, 1998.

Bergman, E. M.; Sieben, J. M.; Smailbegovic, I.; de Bruin, A. B.; Scherpbier, A. J. \& van der Vleuten, C. P. Constructive, collaborative, contextual, and self-directed learning in surface anatomy education. Anat. Sci. Educ., 6(2):114-24, 2013.

Clough, R. W. \& Lehr, R. P. Testing knowledge of human gross anatomy in medical school: an applied contextual-learning theory method. Clin. Anat., 9(4):263-8, 1996.

Dans, E. Educación online: plataformas educativas y el dilema de la apertura. En: Cultura digital y prácticas creativas en educación. Monografía en Línea. Rev. Univ. Soc. Conoc., 6(1):2230, 2009. Disponible en: http://rusc.uoc.edu/index.php/rusc/ article/viewFile/26/21

Drake, R. L.; McBride, J. M.; Lachman, N. \& Pawlina, W. Medical education in the anatomical sciences: the winds of change continue to blow. Anat. Sci. Educ., 2(6):253-9, 2009.

Garg, A. X.; Norman, G. R.; Eva, K. W.; Spero, L. \& Sharan, S. Is there any real virtue of virtual reality?: the minor role of multiple orientations in learning anatomy from computers. Acad. Med., 77(10 Suppl.):S97-9, 2002.

Hildebrandt, S. Lessons to be learned from the history of anatomical teaching in the United States: the example of the University of Michigan. Anat. Sci. Educ., 3(4):202-12, 2010.

Huitt, T. W.; Killins, A. \& Brooks, W. S. Team-based learning in the gross anatomy laboratory improves academic performance and students' attitudes toward teamwork. Anat. Sci. Educ., 8(2):95-103, 2015.

Inzunza, O. \& Bravo, H. Computer animation of photographs, an actual contribution to practical learning of human anatomy. Rev. Chil. Anat., 20(2):151-7, 2002.

Inzunza, O. \& Bravo, H. Impact of two software of human anatomy in the performance of the students practical knowledge. Rev. Chil. Anat., 17(2):205-9, 1999.

Inzunza, O.; D'Acuña, E. \& Bravo, H. Practical evaluation in anatomy. Performance of first year medical students in relation to different ways of asking questions. Int. J. Morphol., 21(2):131-6, 2003.

Johnson, J. H. Importance of dissection in learning anatomy: personal dissection versus peer teaching. Clin. Anat., 15(1):3844, 2002.
Jones, D. G. \& Harris, R. J. Curriculum developments in Australasian anatomy departments. Clin. Anat., 11(6):401-9, 1998.

Kish, G.; Cook, S. A. \& Kis, G. Computer-assisted learning in anatomy at the international medical school in Debrecen, Hungary: a preliminary report. Anat. Sci. Educ., 6(1):42-7, 2013.

Lacruz, C.; Moreno, F. \& Carrasquero, W. La informática educativa en educación superior. Daena Int. J. Good Conscienc., 4(1):116-27, 2009.

Lewis, T. L.; Burnett, B.; Tunstall, R. G. \& Abrahams, P. H. Complementing anatomy education using three-dimensional anatomy mobile software applications on tablet computers. Clin. Anat., 27(3):313-20, 2014.

Mayfield, C. H.; Ohara, P. T. \& O'Sullivan, P. S. Perceptions of a mobile technology on learning strategies in the anatomy laboratory. Anat. Sci. Educ., 6(2):81-9, 2013.

Michaelsen, L. \& Richards, B. Drawing conclusions from the teamlearning literature in health-sciences education: a commentary. Teach. Learn. Med., 17(1):85-8, 2005.

Sheremetov, L. \& Vladimir, L. Hacia la nueva generación de sistemas de aprendizaje basados en la web. Comput. Sist., 5(4):25667, 2002.

Tam, M. D.; Hart, A. R.; Williams, S.; Heylings, D. \& Leinster, S. Is learning anatomy facilitated by computer-aided learning? A review of the literature. Med. Teach., 31(9):e393-6, 2009.

Turney, B. W. Anatomy in a modern medical curriculum. Ann. R. Coll. Surg. Engl., 89(2):104-7, 2007.

Vasan, N. S.; DeFouw, D. O. \& Compton, S. Team-based learning in anatomy: an efficient, effective, and economical strategy. Anat. Sci. Educ., 4(6):333-9, 2011.

Dirección para Correspondencia:

M.Sc. Emilio Farfán Cabello

Académico

Departamento de Anatomía Normal

Pontificia Universidad Católica de Chile

Libertador Bernardo O'Higgins 340

Edificio Pregrado de Medicina $8^{\circ}$ piso

Santiago

CHILE

Email: efarfan@med.puc.cl

Recibido : 08-06-2015

Aceptado: $20-10-2015$ 